\title{
El proceso hacia la paridad en América Latina a la luz de una ideología participativa. Propuesta metodológica aplicable a estudios interdisciplinares de género y ciudadanía de las mujeres
}

\author{
The process towards gender parity in Latin America in \\ the light of a participatory ideology. Methodological \\ proposal applicable to interdisciplinary gender studies \\ and women's citizenship
}

Irune Aguirrezabal Quijera

Universidad Autónoma de Madrid iruneaguirrezabal@gmail.com

Cómo citar/Citation: Aguirrezabal, Irune. 2021. «El proceso hacia la paridad en América Latina a la luz de una ideología participativa. Propuesta metodológica aplicable a estudios interdisciplinares de género y ciudadanía de las mujeres». Deusto Journal of Human Rights,No. 7: 127-156. doi: http://dx.doi.org/10.18543/djhr.1894.

Sumario. Introducción. 1. Diseño metodológico, fuentes y técnicas de análisis de la investigación. 2. Propuesta metodológica aplicada a un problema y objeto de investigación: el proceso paritista latinoamericano. 3. Pertinencia y relevancia social de la propuesta metodológica y objeto de análisis. Referencias bibliográficas.

Resumen: Este artículo describe una propuesta metodológica elaborada para abordar investigaciones vinculadas a estudios de género y, en particular, sobre la ciudadanía de las mujeres. La metodología se enfrenta a los problemas de investigacion desde una aproximación interdisciplinar, integral y circular, vinculando el cuestionamiento que impulsa el pensamiento teórico feminista sobre nociones y paradigmas tradicionales del orden social, económico y político con la praxis, con las tensiones y sinergias estratégicas que se producen en contextos diferenciados. El artículo muestra su aplicación a una investigación ya realizada sobre el proceso hacia la paridad en América Latina. Los dos elementos metodológicos que singularizan el análisis crítico en el que se basa la metodología son: (1) un diálogo circular, con un cuestionamiento constante entre la perspectiva analítica conceptual y la contextual (que en la investigación a la que ha sido aplicada resultó en una estructura tridimensional 
del fenómeno descrito: conceptual, evolutiva en el tiempo y espacio, y del progreso que surge a partir de la tensión entre acción-resistencia); y (2) el amplio y diverso repertorio de fuentes filosóficas, comentadas y participativas, originadas por los mismos actores involucrados en el fenómeno analizado, el «proceso paritista latinoamericano», a la luz de una ideología participativa. La metodología dirigida a investigaciones académicas persigue resolver problemas reales, de ahí que, junto a una metodología cualitativa crítica, en la que se analizan fenómenos, causas, contextos y actores, se propone, además, formular un enfoque prescriptivo, con ánimo propositivo.

Palabras clave: metodología interdisciplinar, ideología participativa, estudios de género, democracia paritaria, América Latina.

Summary: This article presents a methodological proposal developed to address research studies linked to gender studies and, in particular, women's citizenship. The methodology addresses research problems from an interdisciplinary, integral and circular perspective, linking the critique of traditional notions and paradigms of the social, economic and political order by feminist theoretical thought with praxis. That is, with the tensions and strategic synergies that occur in different contexts. The article shows its successful application to a study already carried out on the process towards gender parity in Latin America. The two methodological elements that distinguish the critical analysis on which the methodology is based are: (1) a circular dialogue, with a constant interrogation of the conceptual and the contextual analytical perspectives. In the research to which it has been applied, it resulted in a three-dimensional structure of the phenomenon described: conceptual, evolutionary in time and space, and of the progress arising from the tension between action-resistance; and (2) the wide and diverse repertoire of philosophical, commented and participatory sources, originating from the very actors involved in the phenomenon analysed (the "Latin American paritist process") in the light of a participatory ideology. The methodology intends to help academic research studies by trying to solve real problems; hence, together with a critical qualitative methodology, in which phenomena, causes, contexts and actors are analysed, a prescriptive approach is also formulated, in a propositional spirit.

Keywords: interdisciplinary methodology, participatory ideology, gender studies, parity democracy, Latin America. 


\section{Introducción}

El presente artículo se propone describir una propuesta metodológica para investigaciones que abordan fenómenos o temáticas diversas desde la perspectiva que ofrecen los estudios de género. El artículo describe dicha propuesta metodológica vinculándola a un objeto de investigación concreto al que ya ha sido aplicado con éxito: el proceso de reivindicación de la ciudadanía de las mujeres y hacia la paridad político electoral y la democracia paritaria en América Latina (Aguirrezabal 2020).

La propuesta metodológica se propone por su aportación a los estudios de género, en general, y, de manera particular, cuando se indaga en factores, causas, elementos o actores vinculados a la reivindicación por la ciudadanía de las mujeres. La metodología propuesta sirve para desarrollar un análisis que vincula un debate crítico conceptual, tal y como plantea la teoría política feminista al cuestionar de manera permanente conceptos y paradigmas tradicionales, y el análisis cualitativo crítico del fenómeno concreto objeto de estudio, mediante la combinación e interrelación de distintas disciplinas, y exponiendo el análisis a un cuestionamiento y debate que permita proponer nuevas demandas, conceptos o estrategias. De este modo, la metodología propuesta permite mantener un cuestionamiento permanente a partir del análisis que vincula ambos enfoques, conceptual y contextual, interconectando ambos de manera circular, y dotando de una explicación integral al fenómeno estudiado.

Así, en la investigación a la que ha sido aplicada, partiendo del análisis crítico que realiza el pensamiento feminista sobre la evolución del significado de la categoría política «mujeres», se revisan y discuten los paradigmas y conceptos de la teoría política del Estado sobre la noción de ciudadanía política y social, a la vez que se cuestiona el lugar que ocupan las mujeres en la conceptualización y evolución del Estado Liberal hacia el Estado de bienestar ${ }^{1}$. A través de la metodología circular propuesta, tras un análisis exhaustivo de los conceptos de igualdad sustantiva, paridad y democracia paritaria, se estudia el viaje ${ }^{2}$ de estas nociones entre Europa y América Latina, en contextos diferenciados, así como el viaje interno de esos conceptos entre el marco jurídico y discursivo político en América Latina, desde el nivel regional donde predomina el soft law de los acuerdos internacionales, hasta el nivel

1 Sin perjuicio de la diversidad de modelos (Esping-Andersen 1993, 223).

2 Se utiliza la expresión de "circulación de conceptos» o «conceptos viajeros» en humanidades (Bal 2009). 
estatal, donde se ubican y ponen a prueba en la praxis las leyes de cuotas y paridad adoptadas por los países latinoamericanos. La teoría política feminista y la movilización de las mujeres en red están en todo el proceso analizado. Este análisis previo resulta esencial para avanzar en la comprensión del fenómeno estudiado, las causas, características, los actores, factores, alrededor de la amplia movilización de mujeres en red -y otros actores vinculados- que están detrás del proceso ideológico que lucha por la representación política de las mujeres, a la vez que se discuten los avances ante las resistencias, los límites y las tensiones que son identificadas.

El análisis conceptual y contextual, entrelazado, revisa y cuestiona, en un diálogo permanente, esa movilización de las redes de mujeres en términos estratégicos, presentando las divergencias entre las aproximaciones en Europa, en el ámbito regional latinoamericano y en las legislaciones nacionales, para así exponer las deficiencias actuales y poder identificar las transformaciones pendientes hacia ese horizonte de democracias paritarias que América Latina se ha fijado.

Para desarrollar un análisis circular e integral como el expuesto, la propuesta metodológica descansa en una estrecha relación entre las fuentes utilizadas y los actores analizados. Fruto de ella se origina un proceso asimilado a una ideología ${ }^{3}$, eje en torno al cual gira la propuesta y que, en relación con el problema y objeto específico al que ha sido aplicado y que se recoge en estas páginas, se ha denominado «proceso paritista latinoamericano» 4 .

Un aspecto clave de la metodología propuesta, siguiendo con modelos de investigaciones de movimientos sociales, reside en la interdisciplinariedad como fundamento de las investigaciones en programas de estudios de género.

El interés por los estudios de género, por el feminismo y, en particular, por la temática de la paridad y de las cuotas justifica proponer una propuesta metodológica de investigación que permita obtener una perspectiva más compleja y ambiciosa en una temática de actualidad y relevancia social, sobre todo en América Latina (Aguirrezabal 2020). La incorporación de las mujeres a la política, al ámbito público, a los poderes ejecutivo, legislativo, judicial y electoral, y en todos los niveles de gobernanza territorial, concierne a todos. Tiene una transcendencia electoral, tiene un impacto directo en el

3 Se ha tomado como referencia la teorización sobre las tres tipologías de ideologías desarrollada por Román-Zozaya (2008, 111-132).

4 Véase la explicación sobre la expresión «proceso paritista latinoamericano» en la descripción del problema y objeto de la investigacion a la que ha sido aplicada. 
reparto del poder político. Con cada nueva elección, gracias a la legislación de cuotas y de paridad, los parlamentos van a contar con más mujeres candidatas y más mujeres electas. La participación política de las mujeres implica también cambios en las dinámicas del poder y de las organizaciones políticas, fundamentalmente en los partidos políticos, un espacio masculinizado por excelencia donde antes solo había hombres. Pero, además, la participación paritaria en la toma de decisiones pretende generar efectos catalizadores en la agenda política que coadyuven a erradicar los factores estructurales de la desigualdad que sufren las mujeres. De la representación paritaria sustantiva derivan cambios trascendentales en la cultura política (Pitkin 1967). Además, la propuesta metodológica resulta pertinente para estudiar el movimiento feminista, analizado como movimiento social y político, con impacto en la agenda global, a través del feminismo institucional.

En las líneas que siguen se describe la propuesta metodológica aplicada al objeto de investigación concreto, de ahí que el artículo se divida en tres puntos. El primero de ellos describe el diseño metodológico propuesto y las técnicas de análisis y fuentes utilizadas. El punto 2 aborda el problema y objeto de la investigación concreto al que se ha aplicado dicha metodología. El punto 3 justifica la pertinencia y relevancia social de enfoque metodológico con relación a los problemas que quiere resolver.

\section{Diseño metodológico, fuentes y técnicas de análisis de la investigación}

Tal y como se ha señalado, nos proponemos compartir el diseño metodológico aplicado ya a una investigación, con la esperanza de que dicha propuesta metodológica resulte de interés para aquellas investigaciones que pretenden analizar cómo afecta la intersección de género en fenómenos y temáticas diversas. En el caso concreto que se va a exponer, se ha aplicado al análisis del proceso movilizador de las mujeres en red hacia la paridad política en América Latina. El diseño metodológico se ha propuesto, de manera consciente, dotar de una mirada integral y transversal al fenómeno analizado, en coherencia con la vindicación de la teoría política feminista de la transversalización de género en todas las dimensiones, así como con la agenda transversal e integral del desarrollo sostenible (Naciones Unidas 2015).

De ahí que la metodología se fundamente en la interdisciplinariedad aplicada a los estudios de género. Esa interdisciplinariedad es necesaria cuando el proceso investigador persigue la búsqueda de soluciones 
a problemas reales de investigación; la metodología, las fuentes, las herramientas y técnicas de análisis se articulan en un proceso dinámico que integra el conocimiento desde distintas disciplinas propiciando la integralidad en el análisis, a fin de hallar propuestas de superación del statu quo. La categoría analítica de género permite desarrollar un planteamiento integral crítico del orden social, político, legal y/o económico en el que se inscriben los fenómenos que se abordan de modo que se pueda superar la construcción estructural de la división de género. Hay una motivación propositiva de búsqueda de soluciones. Igualmente, la interdisciplinariedad se vincula con la necesidad de dotar al análisis de los estudios de género de una visión holística desde una perspectiva sistémica. Se quiere superar el enfoque convencional de división temática en silos por un enfoque de sistemas. Esta visión integral y holística, a su vez, se enmarca en la agenda internacional que ha promovido el feminismo institucional, por la cual la propia Agenda 2030 de Objetivos de Desarrollo Sostenible de las Naciones Unidas asume que los 17 objetivos se interrelacionan intrínsecamente entre sí, formando un marco indivisible que tiene por objeto lograr la sostenibilidad holística y coherencia de políticas que se persiguen ${ }^{5}$.

Así, la interdisciplinariedad resulta imprescindible en relación con el objeto de análisis y problema concreto señalados. Al aplicar una perspectiva de género como categoría analítica, no basta con una investigación dedicada a indagar en el proceso hacia la paridad desde una perspectiva de los avances que ofrece el derecho internacional público y su adaptación a la región y a los países. Si bien este enfoque jurídico sobre el derecho a la participación política de las mujeres es ineludible, por sí solo no aporta suficientes elementos de análisis para comprender el fenómeno estudiado. Ha sido necesario acudir a las ciencias sociales para explicar cómo interactúan los actores en el proceso jurídico y político y cómo se ha forjado el movimiento social de mujeres en red que promueve la legislación paritaria en esa región. Del mismo modo, para garantizar una visión global resultaba indispensable indagar en la filosofía del derecho y en la teoría política del Estado para cuestionar conceptos; ha sido igualmente necesario revisar las políticas públicas que promueven la representación paritaria; así como cuestionar las estrategias y acciones que impulsan que la

${ }^{5}$ Las interconexiones entre los 17 ODS y las 169 metas de la Agenda 2030 son ampliamente reconocidas. Ello está permitiendo avances muy interesantes para una implementación de la agenda coherente con el desarrollo de metodologías que permitan visualizar y aplicar las interconexiones entre políticas. Véase, https:// sdginterlinkages.iges.jp/methodology.html 
paridad como herramienta logre el efecto catalizador que modifique los roles de género. A través del enfoque interdisciplinar de los estudios de género se desafía la división tradicional de disciplinas de análisis. La interdisciplinariedad permite ampliar abordar un análisis cualitativo crítico sobre los fenómenos en su plenitud. Así, aplicado al objeto de análisis señalado, se abordan tanto las causas de una discriminación sistémica hacia las mujeres que subordina el ejercicio y titularidad de la ciudadanía, como los efectos catalizadores de las transformaciones en marcha. Y ello mediante un análisis integral a partir del estudio de normas, proyectos, actores, estrategias y conceptos en relación con el proceso movilizador hacia la paridad en esa región concreta.

Desde la perspectiva metodológica se destacan dos aspectos. El primero y más relevante, se refiere a que se trata de una metodología de investigación cualitativa crítica, en la que se analizan fenómenos, causas, contextos y actores, y en la que se cuestiona la construcción conceptual de la paridad bajo las condiciones que ofrece el contexto latinoamericano. Por tanto, predomina una metodología cualitativa que busca, sobre todo, enfrentar los problemas. En segundo lugar, se ha querido además aportar un enfoque prescriptivo, con ánimo propositivo mediante la formulación de una teoría de cambio. A pesar de constituir un enfoque ciertamente menos común en el ámbito académico, forma parte de la metodología utilizada y obedece a su pertinencia en estudios de género, pues se aborda un problema real que requiere soluciones en un mundo cambiante.

En primer lugar, el problema de una investigación de estudios de género obliga a indagar en una comprensión desde un análisis cualitativo crítico ${ }^{6}$ de la complejidad de los hechos, las normas, las estructuras y los procesos en su totalidad, abarcando una mayor comprensión de la complejidad del proceso analizado (Morin et al. 2003). Como se ha dicho, la metodología implica, además, que las investigaciones sigan un proceso de análisis a través de elementos que conduzcan a interpretar los eventos y acontecimientos en el ámbito de las ciencias políticas y sociales, sin perjuicio del análisis jurídico, en su caso.

Así, analizar el papel de los movimientos de mujeres y de la teoría política feminista como actor social y político, pero también como propulsor de nuevos paradigmas teóricos resulta crucial para desarrollar

6 Véase Minayo (2010), quien parte de la distinción entre investigación de ciencias empírico-analíticas, histórico-hermenéuticas y ciencias orientadas críticamente o denominadas ciencias sistemáticas de la acción (a partir del estudio que hace de la fundamentación elaborada por Habermas). 
los conceptos, analizar su evolución y desarrollo, incluido a través del progreso legislativo y de su implementación en políticas públicas. Esos movimientos teóricos y activistas han ido entretejiéndose en un proceso acumulativo ${ }^{7}$, a base de intercambios entre redes de mujeres y de superar las trabas y trampas que van surgiendo frente a nuevas demandas. Y frente a que no se resuelvan las consecuencias de aceptar dichas demandas. $Y$ todo ello mientras las idean van permeando en el feminismo institucional y de Estado (Lovenduski 2005). Más allá de las diferencias sociales, económicas, ideológicas o étnicas que puedan tener las mujeres de la región, la reivindicación por la ciudadanía de las mujeres y por el consecuente reparto del poder entre varones y mujeres se ha concebido como un marco cognitivo $^{8}$ que unifica a las mujeres en su propósito de ruptura con la persistente estructura androcentrista.

Al configurar de tal modo las movilizaciones de las mujeres se ha asimilado a un proceso ideológico, tomando el análisis que realiza Carolyn Román-Zozaya (2008). Su estudio ha servido para argumentar que una ideología es potencialmente dinámica, está orientada a la acción, es multidimensional, contiene un marco de conceptos creados por los productores en un contexto, que a su vez se adapta a los consumidores a través de un marco de relaciones interactivas. Así, al buscar comprender el proceso que se moviliza hacia la paridad en América Latina (como luego se analiza), caracterizado por un factor multidimensional de conexiones entre actores diversos, se han estudiado la amplia diversidad de acciones involucradas, las ideas, los recursos, los proyectos, las personas, las organizaciones y las instituciones que han participado en el desarrollo y expansión del proceso hacia la paridad, a la luz de una ideología.

La referencia a un proceso que se asimila a un proceso ideológico o una ideología ${ }^{9}$ se ha justificado en base a la teorización sobre las tres tipologías de ideologías desarrollada por Román-Zozaya (2008): filosófica, comentada y participativa. La autora analiza cómo estas tres tipologías están creadas respectivamente por filósofos, por intelectuales

7 Se refiere a un factor de acumulación de avances (y sus retrocesos), para explicar la evolución de los pasos hacia la paridad en la región (Bareiro y Soto 2015).

8 Se alude al concepto de marco cognitivo desarrollado por Barbara Hobson (1996).

9 Hace décadas se discute el fin de la era de las ideologías con autores como Raymon Aaron, Daniel Bell o Francis Fukuyama, como culminación de un proceso de la humanidad en el que se impone el liberalismo capitalista democrático. En ese proceso y frente al debate izquierda/progresista-derecha/conservador, irrumpe el feminismo, con una fuerza que puede ser asimilada a una ideología, según la definición acuñada por Faye y García-Borrón (1998) de ideologías como vehículo de grandes movimientos sociales y de pensamiento. 
públicos, que comentan una situación política específica, y por los políticos, inmersos en los procesos políticos formales bajo condiciones de tiempo real.

Frente a una explicación meramente causal ${ }^{10}$, la triple tipología de ideologías sirve para recurrir a la amplia diversidad de fuentes atendiendo justamente al complejo marco de interrelaciones entre agentes productores de avances hacia la paridad. Y, a su vez, a cómo todo ello conduce a una expansión y evolución conceptual, pues se van adaptando los conceptos como si se tratara de la gestación y extensión de una «ideología por la paridad».

Así, tomando la teorización sobre la ideología participativa y las ideologías filosófica y comentada de Román-Zozaya, el análisis realizado eleva la conceptualización de la paridad a la categoría de ideología entendida como un marco complejo multidimensional que se encuentra: 1) afectado por la interdependencia entre conceptos (en el caso analizado, género, igualdad, democracia, ciudadanía, poder, política, paridad, democracia paritaria); 2) producido por "agentes» múltiples y diversos (movimiento feminista y redes de mujeres diversas con apoyo/interacción institucional, tanto de ámbito nacional como regional, así como por parte de organismos internacionales) ${ }^{11}$; y 3) sujeto a la aceptación de los «consumidores» (la ciudadanía latinoamericana, así como los grupos políticos en los parlamentos, los gobiernos, los mismos partidos políticos y otros agentes, que junto a la categoría propiamente de consumidores, a su vez se constituyen en agentes de cambio o resistencias a esa misma ideología paritista); y 4) todo ello en un contexto histórico, político, económico y social, cambiante por definición (Cirillo, 2002), ya que la ideología es un producto social, y por ello, es potencialmente dinámica, y está sujeta a cambios.

10 La autora señalada parte del análisis sobre teoría política e ideologías que realiza Michael Freeden (1996).

11 En el estudio sobre los actores que promueven la paridad en América Latina, que se recoge en la investigación doctoral aludida, se analiza la rotación de las mujeres en ámbitos y redes diferenciados, desde mujeres en redes feministas, a mujeres que participan en los mecanismos gubernamentales de la mujer, mujeres académicas, mujeres que participan de la formulación de políticas públicas promovidas desde organismos regionales o internacionales, entre otras. Esta rotación de mujeres en dichos espacios e instituciones sugiere que, necesariamente, debe tener un impacto el proceso de «producción» de la conceptualización de la paridad como ideología, puesto que se construye, propaga, expande y divulga en todos ellos, con las mujeres en red como nexo (Aguirrezabal 2020). 
Las ideologías fracasarían si no fueran capaces de persuadir y convencer a otros para que actúen de acuerdo con la ideología misma. La conceptualización de la paridad en América Latina, su extensión e implantación en la región no es un producto aislado. Su desarrollo depende de la capacidad de interacción de las ideas que la promueven con el ámbito institucional en las que esas ideas se desenvuelven. La institucionalidad latinoamericana, en connivencia con las redes de mujeres, ha aceptado promover debates sobre la ciudadanía de las mujeres. Y ha sido persuadida en términos generales (no en todos los países, pero casi) de la necesidad de aprobar disposiciones legislativas que garanticen por ley una mayor participación política de las mujeres.

El análisis del proceso paritista como una ideología se fundamenta también en la dinámica regional de interrelaciones entre los agentes productores de dicha ideología paritista que ha ejercido una influencia activa en el desarrollo de la formulación conceptual y estratégica hacia la paridad. El nexo que une a esos agentes en una comunidad en red, lingüística e histórica son las mujeres. Se trata de mujeres filósofas o teóricas feministas, académicas, representantes y expertas de diversos organismos públicos (de organizaciones de la sociedad civil, de organismos internacionales o regionales, entre otros), así como de representantes del ámbito político e institucional (en su mayoría, mujeres políticas). Los mismos agentes productores de esa ideología son, justamente, las fuentes que han servido para registrar, analizar e interpretar datos, discursos, leyes, comentarios en seminarios, publicaciones, resoluciones, declaraciones, proyectos, estrategias u otras fuentes utilizadas en esta investigación.

De este modo, se entiende el proceso paritista desde la categoría de una ideología participativa, producida por representantes del ámbito político. Pero no solo, porque a la vez, está mediatizada por su vinculación e interacción con intelectuales y activistas feministas y, por lo tanto, tiene componentes de ideología comentada. Y, además, se nutre de la (ideología) razón teórica y filosófica (y ello tanto de las propias autoras latinoamericanas, como de otras fuentes, sin duda, norteamericanas y europeas, en particular, españolas ${ }^{12}$ ).

12 Una de las líneas de investigación que surgieron a partir de la investigación aludida se propone indagar en si filósofas, activistas y políticas españolas han podido tener un impacto en el proceso paritista latinoamericano en torno a los años 20052009, cuando coincidiendo con la dinámica generada por la plataforma creada por la Fundación Carolina y los múltiples intercambios de ideas y propuestas entre feministas latinoamericanas y españolas se producen avances en América Latina, a partir de la aprobación del Consenso regional de Quito de 2007, que formula la definición amplia de paridad y el principio transversal de igualdad sustantiva (CEPAL 2007). 
Junto a esta metodología de análisis que vincula la interacción entre las tres tipologías de ideologías, se ha aplicado la teoría de la circulación de ideas en humanidades (Bal 2009). A través de ella se puede explicar la difusión de ideas entre contextos diferenciados (en el caso que se expone entre Europa y América Latina), así como entre los ámbitos regional y nacionales. Incorpora perspectivas de análisis que expliquen cómo se adaptan los conceptos y estrategias a contextos diferenciados, elemento clave que determina, en la investigacion aludida, la esencia de la complejidad del proceso paritista en América Latina. Junto a ello, al analizar el contexto latinoamericano, una región con una fuerte cultura patriarcal e inequidad social y económica se identifican las múltiples trabas y dificultades que se va encontrando la expansión de esa ideología. Para analizar cómo se resuelven, se ha acudido a las propuestas sobre las denominadas tensiones creativas que se exploran y explican los avances en el estudio de los derechos humanos en la globalización (Maquieira 2008, 61-74).

En segundo lugar, pese a ser secundario, el diseño metodológico propuesto desarrolla un análisis prescriptivo mediante la formulación de elementos propositivos. En la investigación aludida se ha propuesto una una teoría de cambio. Se justifica dicho enfoque prescriptivo en una motivación por aportar una nueva formulación estratégica en el proceso que permita avanzar de la estrategia dirigida hacia la paridad a una estrategia más ambiciosa hacia la democracia paritaria. La motivación, a su vez, viene precedida por el hecho de haber desarrollado una labor profesional en un organismo dedicado a promover la igualdad de género, lo que ofrece oportunidades de conocimiento práctico singulares ${ }^{13}$. La experiencia en el terreno, en sedes parlamentarias, el conocimiento práctico sobre acciones y proyectos de diversos organismos regionales e internacionales, la participación en seminarios, reuniones, en algunos de los eventos y debates sobre algunas de las normativas estudiadas, entre otras, ha constituido un elemento facilitador a la hora de interpretar y valorar la diversidad de fuentes recopiladas, muchas de ellas de soft law ${ }^{14}$, que se han podido recopilar. Este enfoque prescriptivo en una

13 Como asesora regional política para América Latina y el Caribe de ONU Mujeres (2013 a 2017).

14 Existen distintas definiciones de este término. Del Toro (2006) utiliza la expresión inglesa soft law, introducida dentro de la terminología jurídica internacional por Lord McNair, para definir aquellos actos, conductas o fenómenos jurídicos caracterizados por carecer de fuerza vinculante, no carentes de efecto jurídicos, o al menos con cierta relevancia jurídica, y sin perjuicio de la fuerza política que puedan tener. 
investigación esencialmente académica enlazaría con el interés de que las políticas públicas se basen en el denominado "conocimiento inteligente» (Innerarity 2011), de modo que las investigaciones en estudios de género dirigidas a la transformación de roles que persigue el feminismo de la igualdad, sirvan para generar sinergias entre el proceso de conocimiento científico y en el proceso de planificación estratégica (Godet 2000). De tal suerte que la aportación práctica de la propuesta metodológica pueda contribuir a orientar la estrategia y acciones más adecuadas para el logro de la Agenda 2030 de los Objetivos de Desarrollo Sostenible; en particular, la meta 5.5., asegurar la participación plena y efectiva de las mujeres y la igualdad de oportunidades en el liderazgo a todos los niveles decisorios de la vida pública, política y económica. Así, la formulación estratégica elaborada encaja con la aspiración a una sociedad con un desarrollo sostenible en América Latina, donde se transversalice el principio de igualdad sustantiva. Y con un modelo de Estado del bienestar, ausente o muy deficitario hoy en la región, que incorpore estrategias hacia la ciudadanía social de las mujeres, puesto que solo así la ciudadanía política puede ser efectiva (Lister y Campling 1997). Por tanto, se trata de una propuesta para que las mujeres sean efectivamente humanas (Facio 2011), hacia la ciudadanía plena de las mujeres.

Otro elemento constitutivo de la propuesta metodológica ha consistido en la interrelación entre la diversidad de fuentes, tradicionales y más innovadoras, con una importante aplicación del soft law y de técnicas de investigación vinculadas a entender cómo interactúan y participan los movimientos sociales. La metodología se basa en un acto deliberado y consciente de búsqueda de fuentes a través de una multiplicidad de recursos bibliográficos, partiendo del interés expuesto en las interacciones, cruces, tensiones y contradicciones entre la pluralidad de actores y acciones presentes en la construcción del «proceso paritista latinoamericano».

Los medios de producción (las fuentes de la investigación) que han desarrollado esta ideología paritista latinomericana compleja y multidimensional coinciden así con la propuesta que recoge la autora Román-Zozaya $(2008,119)$, al exponer y describir las particularidades de cada tipología. A partir de la tabla original que elabora la autora se puede extrapolar que las activistas feministas, políticas y representantes de organismos diversos contribuyen a la ideología comentada, utilizando textos no académicos, manifiestos, journals, cátedras, periódicos, revistas, folletos; por su parte, las mujeres políticas latinas han contribuido a la ideología participativa a través de manifiestos, discursos, folletos, periódicos, medios digitales (facebook, twitter, etc.), 
documentos oficiales de políticas públicas y, por supuesto, a través de disposiciones normativas y leyes. Las expertas politólogas y académicas contribuyen a través de sus contribuciones, que se definirían bajo la tipología de la ideología filosófica.

Las técnicas y herramientas de análisis propuestas son las siguientes:

Primero, herramientas tradicionales de una investigación, basadas en el registro y análisis de los textos, así como la interpretación de estos.

- Análisis de texto y revisión de literatura sobre teoría política y teoría política feminista, acudiendo a autoras europeas y norteamericanas fundamentalmente, buscando un análisis contrastado de opiniones y corrientes, en el espacio, en el tiempo. Análisis de fuentes primarias y secundarias sobre el derecho a la participación política de las mujeres. El análisis se centró en el estudio de las medidas especiales de carácter temporal, las cuotas, así como de la paridad. Con revisión del marco jurídico internacional y regional, así como los marcos normativos de los países, legislación electoral y de los partidos políticos, incluyendo no solo las leyes aprobadas sino también proyectos de ley fracasados. Junto a ello, se hace necesario el análisis de la jurisprudencia más relevante y actual proveniente de tribunales electorales de los países analizados.

- Destaca en el diseño metodológico la utilidad de fuentes del soft law ${ }^{15}$, de modo que se propone acudir a acuerdos y consensos regionales, discursos políticos o resoluciones políticas, de organismos internacionales y regionales, proyectos de ley, declaraciones o acuerdos políticos de los países o discursos políticos, en relación con la materia objeto de investigación.

- Análisis sobre estudios y publicaciones relacionados con la agenda global representativa del feminismo institucional, de organismos multilaterales internacionales, en particular, de

15 Respecto a la inclusión del soft law en la descripción del marco jurídico, resulta necesaria y pertinente, dadas las ventajas y potencialidades del soft law como fenómeno de la sociedad internacional y regional. Constituye una fuente del proceso que se está describiendo. Como ya se ha indicado anteriormente, la autora de este artículo coincide con Del Toro (2006, 513-549), para quien el soft law se incorpora al discurso internacional con independencia de su valor jurídico, de forma que produce ciertos efectos que repercuten de diferentes formas en la formación, desarrollo, interpretación, aplicación y en el cumplimiento del derecho internacional, tanto en el ámbito interno de los Estados como en el propio seno del derecho internacional. 
Naciones Unidas, o de organismos regionales, así como de organizaciones de la sociedad civil, feministas y otras. Además de acuerdos consolidados, estas organizaciones ofrecen material relevante, a través de informes de reuniones preparatorias, planes de trabajo y estrategias de las agencias y organizaciones, campañas de sensibilización, evaluaciones de programas. A través de este se puede analizar la interacción de dichas organizaciones con las redes feministas. La interrelación entre los organismos multilaterales y la sociedad civil global es una fuente en sí misma que sirve para analizar cómo se expande el proceso ideológico (feminismo) en la agenda global.

- En el caso que luego se describe en este artículo (sobre América Latina) se ha realizado un proceso de recopilación, sistematización y análisis de datos sobre la participación política de las mujeres en los diferentes poderes del Estado, en sus distintos niveles de gobierno, así como en los partidos políticos. La recopilación de datos es, a menudo, compleja, dependiendo de los países o del tipo de datos, es un trabajo arduo y supone esfuerzos importantes. Para la sistematización de datos han sido requeridos a menudo ${ }^{16}$ procesos manuales, artesanales, utilizando diversas fuentes, tanto primarias como literatura secundaria, sobre datos no oficiales. El déficit en la sistematización oficial de datos electorales desagregados por sexo constituye una preocupación de los investigadores y activistas en la materia.

Segundo, herramientas basadas en el análisis tras la observación y reflexión que se genera a partir de la participación (escucha, participación u observación) en debates en múltiples espacios de índole tanto política como técnica sobre la materia u objeto de investigación.

La participación en seminarios, las notas de reuniones y la experiencia profesional en organismos, instituciones, redes que abordan investigaciones sobre estudios de género puede constituir un factor muy útil para valorar e interpretar hechos y acuerdos, que ofrece elementos de análisis más profundos y complejos.

Conocer el contexto, las redes de mujeres, los organismos internacionales, el contexto regional, constituye un elemento de

16 Solo el poder legislativo, a través de la Unión Interparlamentaria (UIP) y el Observatorio de Género de la Comisión Económica para América Latina y el Caribe (CEPAL) actualizan los datos regularmente en esa región. 
gran utilidad para comprender e interpretar las fuentes, su valor e importancia en procesos de cambios ${ }^{17}$.

Tercero, se propone acudir a fuentes directas, a través de entrevistas semi estandarizadas a expertas y protagonistas del fenómeno analizado (algo que en el caso de la investigación aludida resultó muy útil).

En el proceso investigador se han entrecruzado estas técnicas, de modo que han ido retroalimentándose mutuamente. Junto a un proceso lineal de conocimiento según evoluciona la investigación, se ha tratado de generar un proceso circular, en el que el apoyo en una técnica ha creado un eslabón dialéctico de un conocimiento posterior. Se ha creado una especie de espiral de ciclos de reflexión, que permitían volver a cuestionar los hallazgos, algunas conclusiones y ahondar más en las tensiones y en sus posibles salidas.

\section{Gráfico 1}

\section{Herramientas y fuentes de la investigación}

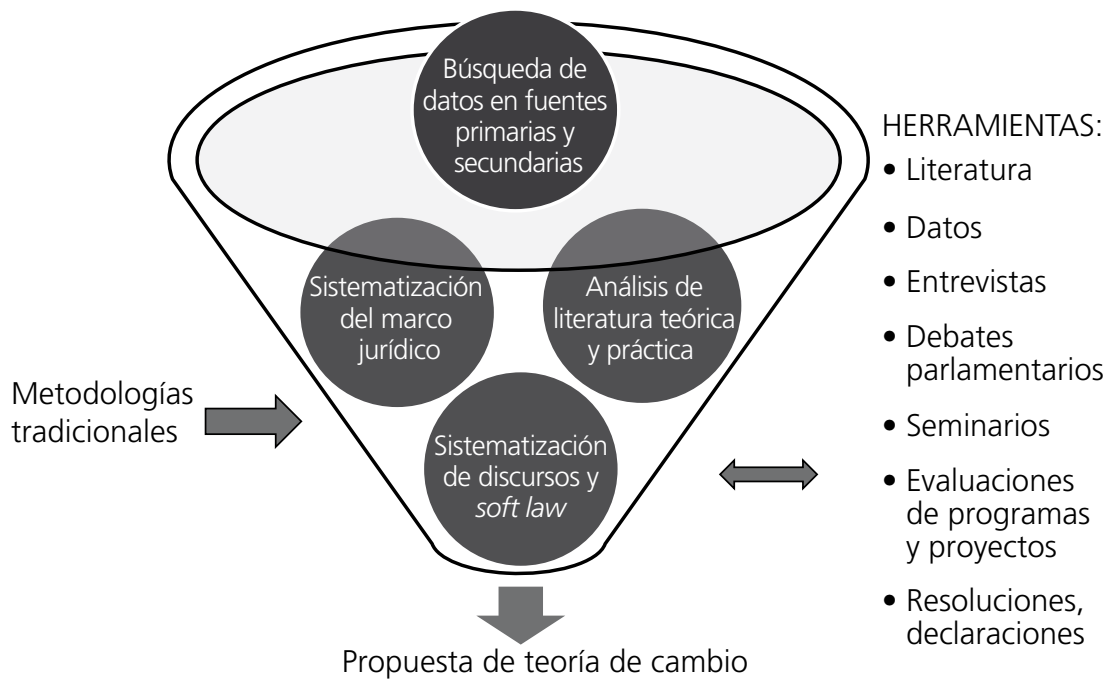

Fuente: Elaboración propia.

17 Así, para entender realmente cómo se ha forjado y qué valor jurídico y político tiene una resolución del Parlamento Latinoamericano y Caribeño, cómo funcionan las redes de mujeres y sus conexiones con las agencias de Naciones Unidas en la región, la experiencia de la autora residiendo durante cinco años en la región ha sido probablemente fundamental. No hubiera sido igual la interpretación de los documentos analizados sin ese conocimiento práctico. 
En suma, a través de esta propuesta metodológica se desarrolla un proceso de aprendizaje constante, que lleva a cuestionamientos a partir de las distintas disciplinas que permitan avanzar más en el análisis para poder vislumbrar soluciones a los problemas que se van encontrando. La interrelación entre las técnicas y fuentes utilizadas y el proceso circular que interconecta los enfoques conceptual y contextual del análisis ha servido para contar con una mirada más profunda y crítica del fenómeno analizado, teórico-filosófica, jurídica, social y política, y ello, además, vinculándolo a la agenda global y al desarrollo del multilateralismo efectivo, impregnado por el feminismo institucional y la reivindicación por la igualdad de género.

\section{Gráfico 2}

\section{Interrelación entre técnicas y metodologías}

\section{RELACIÓN ENTRE LAS METODOLOGÍAS}

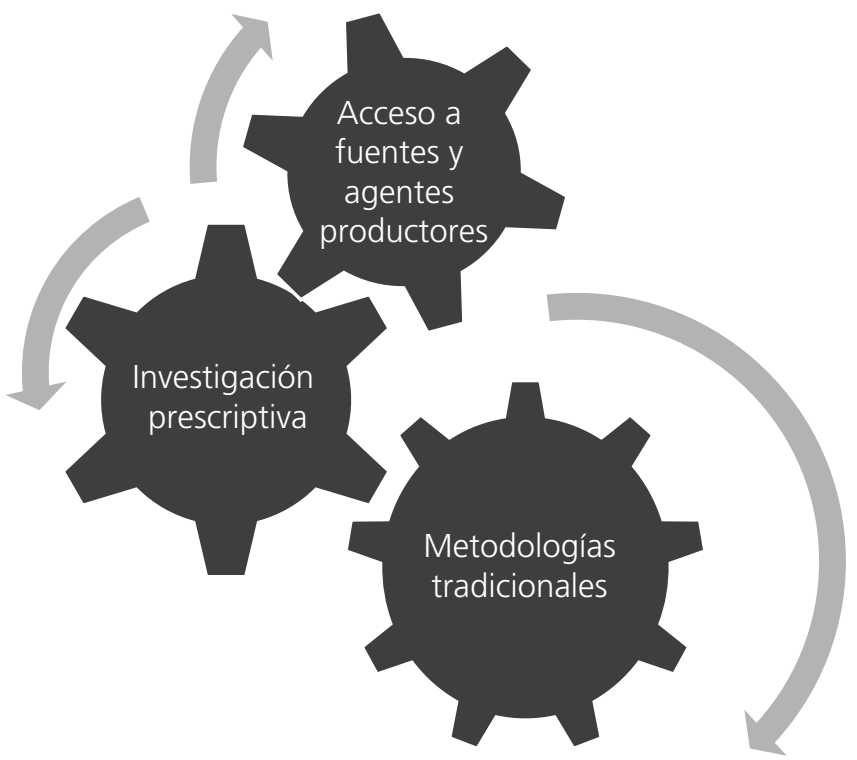

Fuente: Elaboración propia. 


\section{Propuesta metodológica aplicada a un problema y objeto de investigación: el proceso paritista latinoamericano}

La metodología descrita ha sido aplicada ya con éxito a la investigación doctoral (Aguirrezabal 2020) que se viene adelantando y cuyo problema y objeto de análisis resumimos en estas líneas.

Más de 300 años de reivindicación y teorización, a través del pensamiento feminista, han construido un marco conceptual y un lenguaje que han logrado expandirse e impregnar los sistemas normativos e institucionales para avanzar en los derechos humanos de las mujeres. Desde la fundamentación de la teoría política feminista, la investigación aborda la temática de la ciudadanía de las mujeres y la paridad política, interesándose por el proceso de movilización de mujeres en red que ha propiciado el extraordinario desarrollo legislativo a favor de la participación política paritaria en América Latina. Así, el problema científico fundamental que motivó dicha investigación es el interés por dotar de una explicación integral al fenómeno que está detrás del proceso ideológico a favor de la representación política paritaria en América Latina, es decir, sus causas, características, actores y factores. Siguiendo el análisis que realiza Maquieira (2008, 61-74) sobre la tensión entre los derechos humanos y la globalización ${ }^{18}$, se ha desarrollado una investigación los avances de la paridad «como una práctica social y cultural», a fin de explorar cómo, cuándo y porqué la paridad se vuelve significativa para los actores sociales latinoamericanos, así como qué significados se atribuye a la paridad atendiendo al contexto diferenciado de América Latina respecto al europeo.

Así, las dos claves del problema de dicha investigación han consistido en definir el qué y el cómo desde una aproximación integral y circular, aplicando la lógica de la propuesta metodológica descrita. Analizar el qué ha exigido delimitar definiciones en el desarrollo conceptual del denominado "proceso paritista latinoamericano» (del principio de igualdad a las cuotas, a la paridad y, de ahí, a la democracia paritaria). Y ello se ha enlazado con el cómo, que ha requerido conectar las sinergias y las tensiones que se entrecruzan en dicho proceso, en ambos niveles, regional y nacional, en el contexto de América Latina, analizando los distintos actores, las múltiples acciones hacia la paridad, los factores contextuales y las resistencias. De modo

18 Se refiere a la categoría de tensión que se utiliza para la comprensión de los procesos de cambio, en una dialéctica «entre características dinámicas y contrapuestas y su activación en contextos específicos». 
que todos esos elementos puedan explicar el desarrollo estratégico del movimiento de mujeres hacia la paridad como un proceso ideológico, y que, a su vez, reinterpretan y adaptan los conceptos a nuevas estrategias.

Atendiendo a las características de la movilización de mujeres en red, se utiliza a lo largo de la investigación el término de «proceso paritista latinoamericano» (de creación propia). Esta noción permite aludir, no solo al objetivo paritario o de la paridad, esto es, el equilibrio en la representación entre varones y mujeres. Además de ello, el término contempla la multiplicidad de agentes que han participado en su construcción, en referencia a las paritistas francesas que luchan por la paridad a partir de 1992, así como a la sufragista Hubertine Auclert, a quien se alude como primera paritista, o la revolucionaria Olympe de Gouges (Scott 2012). Una expresión con la cual se pretende expresar la fuerza del movimiento de las mujeres latinoamericanas. Por ello, se ha considerado que la movilización paritista tiene características poliédricas y es multifacética. Con ello se alude y analiza no solo el proceso hacia la paridad política entre varones y mujeres, un proceso no unificado, pero con unidad de propósito. Además, la figura del poliedro se refiere a los múltiples agentes y las fuentes de construcción que lo impulsan, de modo que se trata de un proceso complejo, de múltiples caras, social, discursivo, legislativo, académico, político, participativo y transformador. Para realizar dicha investigación, el análisis contextual se debió acotar temporalmente, entre los años noventa y 2019. Partiendo de 1991, momento en el que Argentina adopta la Ley de Cupo, primera medida legislativa en el mundo a favor del avance de las mujeres en política, se focaliza en el punto de inflexión del proceso que se describe, situado en torno a 2007, con la adopción del Consenso regional de Quito (CEPAL 2007). El análisis de datos de mujeres en puestos de representación en distintos poderes y niveles se determinó en 2017 (con una foto fija de ese año). Y el periodo analizado se cierra en 2020, con la incorporación de la legislación de cuotas y de paridad, así como a través del estudio sobre las acciones y resistencias que ha recorrido el proceso hasta ese momento. No obstante, el análisis del enfoque conceptual exigió acudir a fuentes doctrinales anteriores.

El análisis se centró en América Latina, comprendiendo los países denominados latinoamericanos (se excluye Haití). En particular, se centró en diecinueve países: Argentina, Bolivia, Brasil, Chile, Colombia, Costa Rica, Cuba, Ecuador, El Salvador, Guatemala, Honduras, México, Nicaragua, Panamá, Paraguay, Perú, República Dominicana, Uruguay y Venezuela. 
Un aspecto relevante de la investigación fue su enfoque regional, algo que resultó necesario pues se interesaba por los movimientos y redes que se han movilizado en el proceso regional latinoamericano hacia la paridad en la representación política. Un enfoque regional permite observar las tendencias, los compromisos, los discursos políticos y los lineamientos estratégicos que se reflejan a nivel regional a partir del diagnóstico, del marco teórico y estratégico predominante en América Latina. Pero dado que la metodología de la investigación conduce a cuestionar los avances a nivel regional (discursos, acuerdos del soft law mencionado anteriormente) frente a la concreción más limitada de la legislación nacional, fue necesario, además, incorporar un análisis respecto a la situación de los países, en particular, en relación a la legislación, los discursos y datos de mujeres en los distintos poderes y niveles territoriales. Siguiendo con el análisis desde la perspectiva de la interacción de factores sociales en procesos normativos, los diecinueve países comparten elementos comunes. El español (con excepción de Brasil), un elemento clave de conexión y comprensión mutua entre las redes de mujeres en foros regionales. Una base cultural (herencia tanto del colonialismo español y portugués, como del proceso de descolonización y de la tercera ola de democratización. Una reivindicación colectiva por dignificar la categoría mujeres) (Lagarde 2012). Así como un sentido de pertenencia que los identifica como países latinoamericanos (sin perjuicio de las enormes disparidades políticas y económicas o multiculturales entre ellos). La clave regional a través de la cual se desarrolló el análisis fue el movimiento de las redes de mujeres de estos países. Dichas redes comparten, intercambian y movilizan conjuntamente campañas y discursos para generar cambios políticos a nivel regional, que luego se intentan implementar en los países ${ }^{19}$. Igualmente, los gobiernos y parlamentos articulan compromisos regionales que sirven de acicate para lograr avances a nivel nacional.

A modo de síntesis, los cuatro elementos que han singularizado el objetivo de dicha investigación, analizados de manera interrelacionada y que vinculan tanto el enfoque conceptual como el contextual del análisis metodológico propuesto, fueron:

- La necesidad de comprender (y conceptualizar) la definición de la ciudadanía plena de las mujeres (Beltrán y Sánchez Muñoz

19 El potencial transformador y de incidencia política de las redes de mujeres ha sido plasmado a través de los Encuentros Feministas en América Latina y el Caribe desde los años 80 (Álvarez et.al. 2003, 541-575). 
1996) a partir de la problematización del principio transversal de la igualdad sustantiva y del objetivo del empoderamiento de las mujeres. El análisis crítico que hace la teoría política feminista sobre la intersección de democracia, ciudadanía y género exige revisar conceptos y paradigmas de la teoría política del Estado (Valcárcel 1993, 179-184; Puleo 2005), con el propósito de profundizar en la vindicación de desafiar el orden social, económico, político y cultural que sustenta la división de roles de género del patriarcado, como hace el pensamiento feminista, sobre todo, a partir de los sesenta y setenta. Ese análisis sirve para comprender lo que algunas autoras denominan el «proceso de construcción de la ciudadanía de las mujeres en América Latina» (Bareiro 2013), centrado, sobre todo, en los últimos treinta años y que se vincula con las estrategias hacia la paridad. Sin dicho enfoque conceptual, la investigacion se limita a la evolución de acciones, herramientas y estrategias, como desarrollan investigaciones que analizan los marcos jurídicos que promueven la paridad.

— El análisis del «proceso paritista latinoamericano», descrito como una movilización o proceso poliédrico complejo, indagando en las intervenciones de múltiples actores con impactos muy significativos en distintas dimensiones, en el discurso político, en políticas públicas y legislaciones, así como en términos del incremento de la representación de mujeres políticas en la región. Se problematiza el análisis de los actores y de las acciones que han intervenido para promover la paridad en América Latina, fundamentalmente, centrado en los movimientos de mujeres en América Latina, en un sentido laxo (mujeres activistas, académicas, feministas, políticas, juezas). El análisis se amplía a otros actores movilizadores: los organismos internacionales y regionales a favor de la igualdad y del empoderamiento político de las mujeres, analizando su impacto programático, pero también político; actores públicos responsables de adoptar e implementar políticas públicas, esencialmente centradas en legislación paritaria, y previamente en leyes que mandaten cuotas mínimas de mujeres en el ámbito político-electoral. Las fuentes de análisis remiten a esos mismos actores, quienes a su vez dictan nuevas resoluciones, producen publicaciones, promueven políticas públicas, extendiendo el impacto y generando nuevas fuentes.

- La coexistencia de un avance extraordinario en leyes de cuotas y de paridad y de discursos a favor de la representación 
política paritaria junto a notorias brechas que cuestionan la autocomplacencia y muestran la insatisfacción por un resultado deficitario para las mujeres. Por un lado, de los diecinueve países latinoamericanos que fueron analizados, dieciséis países contaban con legislación vigente que incluye un porcentaje de escaños reservados a mujeres que va desde el 20 hasta el $50 \%$. Son: Argentina, Bolivia, Brasil, Chile, Colombia, Costa Rica, Ecuador, El Salvador, Honduras, México, Nicaragua, Panamá, Paraguay, Perú, República Dominicana y Uruguay. Es decir, los tres únicos países que exceptúan la regla de contar con una ley que establece un porcentaje mínimo de mujeres para la representación en el legislativo eran: Cuba (que, sin ley de cuota o paridad, es uno de los países con las tasas más altas de participación de mujeres), Guatemala y Venezuela. De dichos países analizados, ocho (Ecuador, Bolivia, Nicaragua, Costa Rica, México, Argentina, Perú y Colombia) han adoptado leyes denominadas de paridad, en un estricto rango del 50-50 para cada sexo, fundamentalmente, para la composición de las listas electorales (lo que singulariza a América Latina respecto a Europa, pues España o la propia Comisión Europea establecen un reparto equilibrado en un rango de 40/60, en vez del 50/50). Junto a ello, dicha investigacion analiza la extensión del discurso a favor de la paridad en la región, todo lo cual ha producido la triplicación de mujeres parlamentarias en veinte años, pasando de una media regional del 10,7\% a superar el 30\% en 2019, con un estricto 50/50, mujeres y varones. Sin embargo, por otro lado, el proceso hacia la paridad no parece en absoluto homogéneo, de tal forma que, pese a los avances, no se puede concluir que sea satisfactorio. Abundan las brechas en varios $\operatorname{aspectos}^{20}$. Brechas entre el significado y alcance de la paridad plasmada en compromisos políticos y normativos a nivel regional y su materialización efectiva en los países. Brechas en la situación que registran los distintos países latinoamericanos y al interior de cada país, en los distintos niveles territoriales, con enormes déficits en la representación política de las mujeres en niveles sub-estatales y locales ${ }^{21}$ (un pormenorizado estudio del PNUD mostraba que apenas el $11 \%$ de las mujeres llegaban a ser

20 La investigación aludida analiza datos en todas las instancias y poderes del Estado (de la Unión Interparlamentaria y plataformas gubernamentales), así como en los partidos políticos.

21 Un tema del que viene alertando Alejandra Massolo (2002). 
titulares de gobiernos locales) (PNUD 2013), así como en los distintos poderes del Estado. Herramientas como las cuotas y leyes de paridad han incrementado la representación de mujeres en el legislativo, pero aún son muy deficitarios los resultados en el poder judicial y en la justicia electoral22, y en el ejecutivo (aunque en 2014 coincidieron cuatro mujeres como jefas de Estado en sus países, cuatro años después no quedaba ninguna, y en 2017 la media de ministras era del $25 \%)$. Uno de los ámbitos más complejos para las mujeres es el de las organizaciones de los particos políticos, por lo que en las dirigencias de los partidos políticos es donde se produce el verdadero cuello de botella de la paridad sustantiva (Roza, Llanos y Garzón 2010).

- El cuarto elemento se refiere a la dinámica de tensiones y resistencias que debe ser analizada en un diálogo constante que permita evaluar el avance de la representación política de las mujeres $y$, sobre todo, de la democracia paritaria, en el contexto latinoamericano. La región con la mayor inequidad del mundo 23 tiene una evidente necesidad de resignificación de la res pública que contribuya a garantizar los derechos sociales y económicos de las mujeres. A su vez, es una región en la que persiste un sustrato de cultura política y social caracterizada por mantener patrones androcentristas en todas las dimensiones, de manera particular en la política. Entre las resistencias presentes en la competencia político-electoral, uno de los efectos de la tensión se manifiesta en un alarmante incremento de actos de acoso y violencia política hacia las mujeres políticas ${ }^{24}$. El análisis de las resistencias multidimensionales a la ciudadanía plena de las mujeres lleva a cuestionar la efectividad de la paridad en ese

22 Un proyecto desarrollado por las Cortes Supremas de los países de la región, denominado Mapa de género de la justicia, muestra una pirámide con muy pocas mujeres en la cúspide y una base con muchas mujeres en las posiciones más bajas y de menos poder. Véase el mapa de la justicia argentina en: https://om.csjn.gob.ar/ mapagenero/login/mostrarLogin.html

${ }_{23}$ Como reflejan diversas publicaciones, basándose en fuentes del Banco Mundial, 8 de los 10 países que, de acuerdo con su índice de Gini, presentan mayores índices de desigualdad son latinoamericanos. Puede consultarse en https://panampost.com/ mamela-fiallo/2018/07/04/latinoamerica-tiene-8-de-los-10-paises-mas-desiguales-delmundo

24 El interés por este fenómeno creciente se plasma en la amplia literatura regional sobre la materia en los últimos años (Htun y Piscopo 2010, Archenti y Albaine 2013, entre otras). 
contexto ${ }^{25}$, lo que, a su vez, conduce a un análisis crítico sobre las expectativas generadas a partir de las distintas acepciones del concepto de paridad. La representación paritaria está lejos aún de ser una realidad, pero incluso cuando llegan, las mujeres políticas están expuestas a enormes vulnerabilidades y a un alto coste personal. Resurge la vindicación por la ciudadanía social de las mujeres.

Estos cuatro elementos conducen a plantear un desafío conceptual y estratégico ante las diversas nociones del "proceso paritista», que trate de avanzar desde la paridad, a la paridad efectiva, sustantiva y a la democracia paritaria. Si bien es una evidencia que la cultura patriarcal y la desigualdad de género están presentes universalmente ${ }^{26}$, el análisis desarrollado pone énfasis, sin embargo, en la especificidad latinoamericana. Por un lado, a partir de la pujanza extraordinaria del movimiento de las mujeres en red, y su éxito plasmado en la extensión discursiva y legislativa en toda la región y en los países ${ }^{27}$. Por otro lado, a través de la virulencia y gravedad de las resistencias a la paridad, generando tensiones constantes hasta lograr transformaciones profundas.

El análisis crítico revisionista desde la teoría feminista permite afirmar que no cabe disociar la dimensión política de las demás dimensiones de la vida, pues lo importante es contar con recursos, condiciones y oportunidades que promuevan la autonomía de las mujeres, con capacidad y consciencia de elegir (Lister 1997). El análisis desarrollado permitió profundizar en el concepto amplio de

25 Se crea una tipología que permite clasificar y analizar las resistencias a la paridad sustantiva. Por un lado, resistencias ligadas al nudo estructural de la desigualdad, la cultura patriarcal, los déficits de la dimensión económica y social propia de América Latina y los movimientos anti-género; y, por otro lado, las resistencias que se refieren propiamente a la competencia político electoral.

26 Afirmación que se sostiene a partir de todo el andamiaje discursivo y de datos empíricos de organismos internaciones, y que lleva a mantener la exigencia de cumplimiento de la Declaración de Beijing de 1995 y de la Agenda 2030 de Desarrollo Sostenible.

27 La investigación profundiza en dos perspectivas. Por un lado, se indaga en la evolución legislativa de los países desde la adopción de cuotas hasta las leyes de paridad, destacando hitos positivos, como nuevas leyes o sentencias de tribunales electorales, que han permitido avanzar en la representación política de las mujeres pese a los obstáculos, burlas y deficiencias en la legislación. La segunda perspectiva indaga en la expansión del proceso paritista a través de una sistematización de debates y discursos en foros nacionales, incluidos parlamentarios (en un periodo de dieciocho meses), donde se muestra el potencial de expansión y difusión de dicho proceso (ideológico) paritista. 
democracia paritaria latinoamericana, vinculado a la ciudadanía plena de las mujeres y demostrar que es más complejo y ambicioso que el de paridad. Un hilo argumentativo constante de dicha investigación consistía en la necesidad de profundizar en una propuesta estratégica diferenciada a partir de las interpretaciones sobre las nociones de paridad y de democracia paritaria. En la búsqueda de un nuevo contrato social -y sexual- (Pateman 1988), en el que estén incluidas las mujeres (Sánchez Muñoz 2004, 586-604), se encuentra la noción de democracia paritaria latinoamericana, noción que se vincula no solo a la dimensión política de la ciudadanía sino también a la social y económica: la noción genérica de ciudadanía ${ }^{28}$, que incorpora tanto la titularidad como el ejercicio efectivo del conjunto de derechos públicos subjetivos, económicos, sociales, culturales, (status civitatis), con la intersección de género. Una dimensión marcadamente irresuelta en América Latina, donde las mujeres siguen siendo una categoría subordinada, como se demuestra en el análisis de las resistencias, con un amplio sometimiento a la informalidad en el empleo y a su carga de las tareas del cuidado.

Fruto del análisis se propone que, tomando la reivindicación de «lo personal es político» (un concepto que se vincula a muchas autoras, entre otras a Carol Hanish, del movimiento feminista radical, en torno a 1969), el momento actual requiere ahondar en lo social. La vindicación por la ciudadanía plena exige una estrategia integral planteada desde el axioma que se resume en "lo social es paritario y es democrático (democracia paritaria)», porque solo desde la ciudadanía social, desde el ejercicio efectivo de todos los derechos subjetivos, se pueden garantizar los recursos y oportunidades reales para todos y todas $\mathrm{y}$, por ende, se va a lograr que, efectivamente, la paridad política lo sea con efectos sustantivos, para transformar el trasfondo cultural patriarcal. Ello exige que la movilización paritista se asocie, indisolublemente, a una reivindicación más amplia por el principio de igualdad sustantiva como valor y objetivo transversal e integral, esto es, como fundamento del acuerdo de convivencia de la democracia latinoamericana. Ello requiere una estrategia integral para reforzar el Estado de bienestar que garantice la ciudadanía social de las mujeres, y una estrategia integral que conduzca hacia la democracia paritaria latinoamericana. El contexto del post covid-19 pondrá a

28 Véase el análisis sobre la titularidad y el ejercicio de la ciudadanía de Pérez-Luño (2002). 
prueba dicho análisis, evidenciando las deficitarias condiciones de las mujeres en la región con la mayor inequidad del mundo, y ello pese a los avances que irá introduciendo la presencia de las mujeres (Phillips 1995). Las mujeres latinoamericanas son, sin duda, las más afectadas por las graves deficiencias de un sistema que las mantiene como subcategoría. Por ello, frente al mantra del neoliberalismo que se impuso en Latinoamérica hace más de 40 años, quizás esta crisis sea la oportunidad para que el feminismo encuentre alianzas que anhelan también repensar y recuperar los valores del Estado social, asumiendo y distribuyendo responsabilidades que definitivamente sitúen a varones y mujeres como iguales en todas las dimensiones de la vida.

Por último, siguiendo la metodología propuesta, se optó por configurar el análisis desde una estructura tridimensional con tres grandes capítulos, de modo que pudiera enlazar la problematización del proceso que se describe desde tres aproximaciones: primero, conceptual, a fin de analizar si hay disputa o acuerdo sobre los conceptos (de igualdad, ciudadanía, gobernanza, universalismo, paridad, democracia paritaria). En segundo lugar, desde la perspectiva del desarrollo evolutivo, a lo largo de los veinte últimos años, sobre todo, y a partir de los diferentes espacios (antecedentes en Europa, ámbito regional y de los países de América Latina). Y en tercer lugar, desde la dimensión del progreso de las transformaciones, entendido como el resultado de la tensión creativa ${ }^{29}$ que se genera de la dinámica entre acciones y resistencias contextualizadas en la región, y ello desde una perspectiva multidimensional. Esta estructura tiene la finalidad de conectar el análisis conceptual con el contextual, el qué y el cómo, ya descritos, aportando mayor complejidad que el que puede derivarse de un análisis lineal sobre el desarrollo legislativo y discursivo a favor de la participación política de las mujeres en la región analizada. La estructura permite, además, ir acercando a los lectores a la teoría de cambio, a modo de propuesta conceptual y estratégica, que se presenta junto a las conclusiones, cumpliendo con el propósito propositivo que pueden desarrollar las investigaciones de estudios de género.

29 Maquieira $(2008,61-74)$ se refiere a la categoría de tensión para la comprensión de los procesos de cambio, en una dialéctica entre características dinámicas y contrapuestas y su activación en contextos específicos. 


\section{Pertinencia y relevancia social de la propuesta metodológica y objeto de análisis}

La metodología desarrollada tiene como resultado un proceso de investigación multidisciplinar, multinivel, transnacional e integral, desde una búsqueda incesante de capturar las sinergias y tensiones, asimilando la movilización teórica y activista que produce el feminismo y la teoría política feminista a una ideología participativa, a base de utilizar e interpretar un entramado de fuentes diversas, de interrelacionar a los actores y productores de esas fuentes y de contraponer múltiples conceptos entre ellos, de modo que se puedan cuestionar conceptos y estrategias a fin de formular nuevas propuestas que se alineen con la praxis, según los contextos específicos, ya que los conceptos viajan y se adaptan, a la vez que las estrategias para el logro de los derechos humanos deben ir mutando.

La metodología propuesta -y aplicada ya al problema y objeto de investigación descritos en este artículo- se propone contribuir al conocimiento en una temática de gran interés. Ello se manifiesta a través de la vasta e importante literatura sobre la participación política de las mujeres que la teoría política feminista en América Latina ha producido en estos treinta años, con numerosísimas investigaciones para defender las cuotas y la paridad ${ }^{30}$, así como de análisis sobre los obstáculos que perduran y debilitan el ejercicio de la política por las mujeres. Igualmente, existe una interesante literatura sobre los movimientos de las mujeres de la región (Vargas 2002) y numerosas investigaciones sobre el derecho a la participación política ${ }^{31}$.

Se trata de una temática de evidente relevancia social y académica. Cada año nuevos países se incorporan al nuevo escenario político que irá definiendo la participación paritaria en la vida política. En la región de América Latina, en junio de 2020, Perú se incorporó a la lista de países con paridad, vertical y horizontal, en elecciones regionales $y$, en

30 Entre otras, Jones (2000), Bareiro et al. (2004), Archenti y Tula (2007), Ríos (2008), Bareiro y Soto (2015) Archenti y Tula (2014) y Llanos y Martínez (2017).

31 Beatriz Llanos y Marta Martínez (2017) mostraron en una ponencia presentada en el Congreso de Ciencias Políticas de ALACIP (Perú, 2015) que la temática de las cuotas y la paridad ha sido una de las más investigadas por politólogas y expertas en género en la región. El interés por la participación política de las mujeres se refleja en el involucramiento de organizaciones regionales y agencias de las Naciones Unidas mediante estrategias, acciones y publicaciones dedicadas a promoverlo: ONU Mujeres, Programa de Naciones Unidas para el Desarrollo, Comisión Interamericana de las Mujeres, ParlAméricas, Banco Interamericano de Desarrollo, IDEA, entre otras. 
octubre de ese mismo año, Honduras aprobó la paridad con alternancia en diputaciones y alcaldías y primeras regidurías. Resulta pertinente seguir analizando los argumentos y estrategias a favor de la paridad en el sistema electoral por ser esta legítima, justa y beneficiosa para que la agenda pública recoja visiones plurales de la sociedad, pero, sobre todo, porque es la expresión misma de la democracia (Johnson 2000, 69-96).

A nivel internacional, la relevancia de la participación política y de las tensiones que produce el fenómeno de la violencia política se ha puesto de manifiesto de nuevo este año, al tratarse como tema prioritario abordado durante la sesión anual de la Comisión de la Condición Jurídica y Social de la Mujer (conocida en sus siglas en inglés como (SW) en marzo de $202^{32}$.

Por ello, sin perjuicio de que la metodología se dirija a investigaciones académicas, tiene una vinculación directa con la agenda política global que apuesta por un desarrollo sostenible. Se enmarca en el proceso de conceptualización y planificación estratégica de las organizaciones internacionales y regionales en su colaboración con los Estados, para alcanzar la ambiciosa Agenda 2030 que incluye el Objetivo 5, "lograr la igualdad de género y empoderar a todas las mujeres y las niñas», especialmente, la meta 5.5., dirigida a uasegurar la participación plena y efectiva de las mujeres y la igualdad de oportunidades de liderazgo a todos los niveles de la toma de decisiones en la vida política, económica y pública». En suma, la metodología se propone como una aportación a uno de los debates académicos más necesarios para abordar la gran transformación pendiente del S. XXI, la que haga una realidad la democracia feminista (Miyares 2003).

\section{Referencias bibliográficas}

Alvarez, Sonia E., et. al. 2003. «Encountering Latin American and caribbean feminisms». Signs: Journal of women in culture and society. 28, n. 2: 541579.

Aguirrezabal, Irune. 2020. La Democracia Paritaria en América Latina: tres dimensiones explicativas del proceso, tesis doctoral defendida el 10 de julio. Madrid: Universidad Autónoma de Madrid. https://Inkd.in/eTmd8zZ

32 El tema objeto de debate del sexagésimo quinto período de sesiones fue la participación de las mujeres en la vida política, pública y económica y sus efectos en el desarrollo sostenible. Véase UN-Women (2021). 
Archenti, Nélida y Laura Albaine. 2013. «Los desafíos de la paridad de género. Tensión normativa y violencia política en Bolivia y Ecuador». Revista Punto Género 3: 195-219.

Archenti, Nélida y María Inés Tula. 2007. "Cuotas de género y tipo de lista en América Latina», Opinião Pública 13, n. 1: 185-218.

Archenti, Nélida y María Inés Tula. 2014. "Cambios normativos y equidad de género. De las cuotas a la paridad en América Latina: los casos de Bolivia y Ecuador». América Latina Hoy 66: 47-68.

Bal, Mieke. 2009. Conceptos viajeros en las humanidades: una guía de viaje. Murcia: Cendeac.

Bareiro, Line, et al. 2004. Sistemas electorales y representación femenina en América Latina. Serie Mujer y Desarrollo, n. 54. Santiago de Chile: CEPAL.

Bareiro, Line, et al. 2013. La ciudadanía de las mujeres en las democracias de las Américas. Comisión Interamericana de Mujeres (OEA) e Instituto Internacional para la Democracia y la Asistencia Electoral (IDEA). OEA/Ser.LII.6.12.

Bareiro, Line y Lilian Soto. 2015. La hora de la igualdad sustantiva: Participación politica de las mujeres en América Latina y el Caribe Hispano. México: ONU Mujeres.

Beltrán, Elena y Cristina Sánchez Muñoz (eds). 1996. Las ciudadanas y lo político. Madrid: Instituto Universitario de Estudios de la Mujer. Universidad Autónoma de Madrid.

CEPAL. 2007. Décima Conferencia Regional sobre la Mujer de América Latina y el Caribe. Consenso de Quito. Accedido el 20/11/2020. https://www.cepal. org/es/eventos/decima-conferencia-regional-la-mujer-america-latina-caribe

Cirillo, Lidia. 2002. Mejor huérfanas: por una crítica feminista al pensamiento de la diferencia (Vol. 121). Barcelona: Anthropos.

Del Toro, Mauricio I. 2006. El fenómeno del soft law y las nuevas perspectivas del derecho internacional. Anuario mexicano de derecho internacional, 6, 513-549. México D.F.: Universidad Nacional Autónoma de México.

Esping-Andersen, Gøsta. 1993. "Las tres economías políticas del Estado de bienestar». En Desarrollo Institucional y Conflicto. De la geopolitica a la distribución del ingreso, Compilado por Sebastián Mazzuca, 229-265. Buenos Aires: Banco de Desarrollo de América Latina.

Facio, Alda. 2011. "Viena 1993, cuando las mujeres nos hicimos humanas». Pensamiento iberoamericano 9: 3-20.

Faye, Jean Pierre y Juan Carlos García-Borrón. 1998. El siglo de las ideologías. Barcelona. Ediciones del Serbal.

Freeden, Michael. 1996. Ideologies and political theory: A conceptual approach. Oxford: Oxford University Press.

Godet, Michel. 2000. "The art of scenarios and strategic planning: tools and pitfalls». Nueva York. Technological forecasting and social change $65, \mathrm{n}$. 1: 3-22.

Hobson, Barbara. 1996. «Identidades de género. Recursos de poder y Estado de bienestar». En Las ciudadanas y lo político. Editado por Elena Beltrán y Cristina Sánchez, 61-96. Madrid: Instituto Universitario de Estudios de la Mujer. 
Htun, Mala y Jennifer Piscopo. 2010. «Presence Without empowerment? Women in politics in Latin America and the Caribbean», CPPF Working Papers on Women in Politics 2. Nueva York: Conflict Prevention and Peace Forum.

Innerarity, Daniel. 2011. La democracia del conocimiento. Barcelona: Paidós. Johnson, Niki. 2000. «¿Democracia a medias?», Revista Uruguaya de Ciencia Política 12: 69-96.

Jones, Mark. 2000. "El sistema de cuotas y la elección de las mujeres en América Latina: El papel fundamental del sistema electoral». En La democracia paritaria en la construcción europea, coordinado por Paloma Saavedra y Carmen Castro, 35-46. Madrid: Coordinadora Española del Lobby Europeo de Mujeres.

Lagarde, Marcela. 2012. El feminismo en mi vida: hitos, claves y utopías. México D.F.: Inmujeres. Acceso el 15/10/2020. http://repositorio.ciem.ucr. ac.cr/jspui/handle/123456789/121

Lister, Ruth y Jo Campling. 1997. Citizenship: feminist perspectives. Londres: Aardvark Editorial.

Llanos, Beatriz y Marta Martínez. 2017. La democracia paritaria en América Latina. Los casos de México y Nicaragua. Washington DC. Comisión Interamericna de la Mujer.

Lovenduski, Joni. 2005. Feminizing politics. Oxford: Polity.

Maquieira, Virginia. 2008. "Tensiones creativas en el estudio de los derechos humanos en la era global». En Antropología de orientación pública: visibilización y compromiso de la Antropología. Coordinado por Mercedes Jabardo, Pilar Monreal y Pablo Palenzuela, 61-74. San Sebastián: Ankulegi.

Massolo, Alejandra. 2002. «El espacio local: oportunidades y desafíos para el empoderamiento de las mujeres. Una visión latinoamericana». Ponencia presentada en las "Jornadas sobre Género y Desarrollo", Vitoria-Gasteiz: Ayuntamiento de Vitoria-Gasteiz, 23-24 de mayo. Acceso el 15/10/2020. http://webcache.googleusercontent.com/ search?q=cache:RGW1CZsLv_kJ:municipios.unq.edu.ar/modules/mislibros/ archivos/massoloelespaciolocal. $\cdot \mathrm{doc}+\& \mathrm{~cd}=1 \& \mathrm{hl}=\mathrm{es} \& \mathrm{ct}=\mathrm{clnk} \& \mathrm{gl}=\mathrm{es}$

Minayo, Maria Cecilia. 2010. "Los conceptos estructurantes de la investigación cualitativa». Salud colectiva, 6: 251-261.

Miyares, Alicia. 2003. Democracia feminista. Valencia: Universitat de València.

Morin, Edgar, Emilio R. Ciurana y Raúl Motta. 2003. El pensamiento complejo como método de aprendizaje en el error y la incertidumbre humana. Barcelona: Gedisa.

Naciones Unidas. 2015. Objetivos de desarrollo sostenible. Accedido el 20/11/2020. https://www.un.org/sustainabledevelopment/es/2015/09/laasamblea-general-adopta-la-agenda-2030-para-el-desarrollo-sostenible/

Pateman, Carole. 1988. The sexual contract. Redwood City, California: Standford University Press.

Pérez-Luño, Antonio. 2002. "Ciudadanía y definiciones». Cuadernos de Filosofía del Derecho. Doxa. 25: 177-211.

Phillips, Anne. 1995. The politics of presence. Nueva York: Oxford University Press. 
Pitkin, Hanna. F. 1967. The concept of representation. Berkeley: Univ of California Press.

Programa de Naciones Unidas para el Desarrollo (PNUD). 2013. ¿Cuánto hemos avanzado? Un análisis de la participación política de las mujeres en los gobiernos subnacionales en América Latina y el Caribe. Panamá: PNUD.

Puleo, Alicia. 2005. "Lo personal es político: el surgimiento del feminismo radical». En Teoría feminista: de la ilustración a la globalización. Editado por Celia Amorós y Ana de Miguel, 35-67. Madrid: Minerva.

Ríos, Marcela (ed.). 2008. Mujer y política: el impacto de las cuotas de género en América Latina. Santiago de Chile: FLACSO Chile e IDEA Internacional.

Román-Zozaya, Carolyn. 2008. «Participant ideology: A new perspective on politicians and ideology». Journal of Political Ideologies 13, n. 2: 111-132.

DOI: 10.1080/13569310802075944

Roza, Vivian, Beatriz Llanos y Gisela Garzón. 2010. Partidos políticos y paridad: la ecuación pendiente. Washington DC: IDEA y Banco Interamericano de Desarrollo.

Sánchez Muñoz, Cristina. 2004. La ciudadanía social de las mujeres. En Constitución y derechos fundamentales, editado por Jerónimo Betegón, et al. 586-604. Madrid: Secretaría General Técnica. Presidencia del Gobierno.

Scott, Joan. 2012. Parité!: La igualdad de género y la crisis de universalismo francés. Mexico: Fondo de Cultura Económica.

Toro, Mauricio I. del 2006. «El fenómeno del soft law y las nuevas perspectivas del derecho internacional». Anuario mexicano de derecho internacional 6: 513-549.

UN-Women. 2021. Commission on the Status of Women, Acceso el 20/11/2020. https://www.unwomen.org/en/csw/csw65-2021.

Valcárcel, Amelia. 1993. "Sobre revolución y misoginia». Actas del Seminario Permanente Feminismo e Ilustración 1988-92, coordinado por Celia Amorós, 179-184, Madrid: Dirección General de la Mujer.

Vargas, Virginia. 2002. "Los feminismos latinoamericanos en su tránsito al nuevo milenio (Una lectura político personal)». En Estudios y otras prácticas intelectuales latinoamericanas en cultura y poder. Compilado por Daniel Mato, 307-316, Caracas: Consejo Latinoamericano de Ciencias Sociales. 


\section{Copyright}

Deusto Journal of Human Rights / Revista Deusto de Derechos Humanos is an Open Access journal; which means that it is free for full and immediate access, reading, search, download, distribution, and reuse in any medium only for non-commercial purposes and in accordance with any applicable copyright legislation, without prior permission from the copyright holder (University of Deusto) or the author; provided the original work and publication source are properly cited (Issue number, year, pages and DOI if applicable) and any changes to the original are clearly indicated. Any other use of its content in any medium or format, now known or developed in the future, requires prior written permission of the copyright holder.

\section{Derechos de autoría}

Deusto Journal of Human Rights / Revista Deusto de Derechos Humanos es una revista de Acceso Abierto; lo que significa que es de libre acceso en su integridad inmediatamente después de la publicación de cada número. Se permite su lectura, la búsqueda, descarga, distribución y reutilización en cualquier tipo de soporte sólo para fines no comerciales y según lo previsto por la ley; sin la previa autorización de la Editorial (Universidad de Deusto) o la persona autora, siempre que la obra original sea debidamente citada (número, año, páginas y DOI si procede) y cualquier cambio en el original esté claramente indicado. Cualquier otro uso de su contenido en cualquier medio o formato, ahora conocido o desarrollado en el futuro, requiere el permiso previo por escrito de la persona titular de los derechos de autoría. 